\title{
Estimativa da resistência do solo à penetração baseada no índice S e no estresse efetivo
}

\author{
Rui da S. Andrade', Luís F. Stone ${ }^{2} \&$ Sinnara G. de Godoy ${ }^{3}$
}

\begin{abstract}
RESUMO
Objetivou-se, com este trabalho, verificar se a equação proposta por Dexter et al. (2007) para cálculo da resistência do solo à penetração com base na recíproca do índice $\mathrm{S}$ e no estresse efetivo, é aplicável aos solos do cerrado. Para tanto, correlações foram estabelecidas entre os valores calculados e os atributos do solo e comparados com valores críticos estabelecidos na literatura e dados de campo. O estudo foi realizado com 2242 amostras de solo do cerrado, das quais constavam informações sobre classificação textural, densidade do solo e retenção da água do solo. Verificou-se que valores de resistência do solo à penetração iguais ou maiores que $1900 \mathrm{kPa}$, determinados no conteúdo de água do solo equivalente à capacidade de campo, podem ser considerados indicadores de solos compactados e que a equação proposta por Dexter et al. (2007) para estimativa da resistência do solo à penetração é adequada aos solos do cerrado, embora deva ser mais testada para determinar seu poder de predição.
\end{abstract}

Palavras-chave: compactação do solo, densidade do solo, conteúdo de água do solo, textura do solo

\section{Estimation of soil resistance to penetration based on the $\mathrm{S}$ index and effective stress}

\begin{abstract}
This study aims to verify that the equation proposed by Dexter et al. (2007) for calculating the soil resistance to penetration, based on the reciprocal of the $\mathrm{S}$ index and on effective stress is applicable to 'Cerrado' soils. Correlation was established between the calculated values and soil attributes and it was compared with critical values in the literature and field data. The study was conducted with 2242 soil samples of 'Cerrado' soils, which contained information on textural classification, bulk density and soil water retention. It was found that value of soil penetration resistance equal to or greater than $1900 \mathrm{kPa}$, determined at soil water content equivalent to field capacity, can be considered indicator of compacted soils and that the equation proposed by Dexter et al. (2007) to estimate the soil resistance to penetration is adequate for 'Cerrado' soils. However, it should be further tested to determine its predictive power.
\end{abstract}

Key words: soil compaction, bulk density, soil water content, soil texture 


\section{INTRODUÇÃO}

A agricultura praticada nas diversas regiões brasileiras tem propiciado a compactação dos solos, tanto nos sistemas plantio direto e preparo convencional do solo, como em pastagens (Ralisch et al., 2008), o que pode limitar o crescimento das raízes.

A resistência do solo à penetração (RP) é uma estimativa do impedimento mecânico que o solo oferece às raízes, sendo um dos mais comumente citados fatores físicos que afetam o crescimento das raízes (Silva et al., 2008).

A determinação da RP em condições de campo tem sido feita, normalmente, com o auxílio de penetrômetros, que são aparelhos de fácil manuseio e não requerem muito tempo para a tomada das medições (Dexter et al., 2007). Segundo esses autores, nessas medições não são levados em conta a resistência do solo ao cisalhamento, sua compressibilidade nem o atrito do solo com o metal do penetrômetro.

Existe uma estreita relação entre RP, densidade do solo, conteúdo de água, teor de argila e teor de matéria orgânica (Imhoff et al., 2000; Tormena et al., 2004; Ribon \& Tavares Filho, 2004). A relação direta da RP com a densidade do solo é resultado da compactação e degradação da sua estrutura (Busscher et al., 1997); o conteúdo de água do solo influi inversamente na RP devido ao efeito lubrificante da água ao redor das partículas do solo, ou seja, com sua diminuição há incremento em RP (Almeida et al., 2008); o teor de argila afeta a coesão entre as partículas e o de matéria orgânica está relacionado à agregação e à estruturação do solo (Sá \& Santos Júnior, 2005).

Com base nessas interações desenvolveram-se diversos modelos que correlacionam RP e alguns desses atributos do solo. Entre esses pode-se citar os propostos mais recentemente por Silva et al. (2008), que estabelecem relações entre RP e o teor de argila, densidade do solo e conteúdo de água, e por Almeida et al. (2008), que relacionam RP ao conteúdo de água do solo.

Dexter et al. (2007) propuseram, em estudo com solos de clima temperado, um modelo composto por dois termos principais: o primeiro está baseado no grau de compactação e na estruturação do solo e o segundo é atribuído ao conteúdo de água no solo. O primeiro termo é a recíproca do índice $\mathrm{S}$ de qualidade física do solo proposto por Dexter (2004) e o segundo é o estresse efetivo devido à pressão da água dos poros. Conforme esses autores, o modelo proposto é lógico, tem significado físico e é aplicável a todas as classes de solo e texturas, sem nenhuma mudança nos parâmetros da equação.

Whalley et al. (2005) constataram que, sozinho, o estresse efetivo pode ser usado na predição de RP em solos de baixa densidade mas não em solos de alta densidade. A equação proposta por Dexter et al. (2007) vai justamente neste sentido. Em baixas densidades o índice $\mathrm{S}$ é alto e o termo do estresse efetivo domina, contudo, em altas densidades, o índice $\mathrm{S}$ é baixo e o estresse efetivo sozinho não é suficiente para a predição de RP.

Objetivou-se neste trabalho verificar se a equação proposta por Dexter et al. (2007) para cálculo da resistência do solo à penetração é aplicável aos solos do cerrado mediante o estabelecimento de correlações entre os valores calculados e os atributos do solo e de comparação com valores críticos estabelecidos na literatura e dados de campo.

\section{Material e Métodos}

O estudo foi realizado com 2242 amostras de solo, das quais constavam informações sobre classificação textural, densidade do solo (Ds) e retenção da água do solo, registradas nos bancos de dados dos Laboratórios de Solo da Embrapa Arroz e Feijão e Embrapa Cerrados. Essas amostras abrangiam solos sob cerrado das regiões centro-oeste, norte e nordeste do Brasil.

Os dados de retenção da água do solo foram ajustados a uma curva pela equação de Genuchten (1980), que é dada por:

$$
\theta=(\theta \mathrm{s}-\theta \mathrm{r})\left[1+(\alpha \mathrm{h})^{\mathrm{n}}\right]^{-\mathrm{m}}+\theta \mathrm{r}
$$

em que:

$\theta, \theta$ s e $\theta$ r são, respectivamente, os conteúdos de água do solo correspondentes à tensão $\mathrm{h}$, à saturação e à umidade residual, em $\mathrm{kg} \mathrm{kg}^{-1}$

$\mathrm{h}$ - tensão matricial da água do solo, em hPa, n e m (m $=1-1 / \mathrm{n}$ ) são parâmetros empíricos adimensionais de ajuste $\mathrm{e}$ $\alpha$ é um parâmetro expresso em $\mathrm{h} \mathrm{Pa}^{-1}$

$\mathrm{O}$ ajuste foi feito com o auxílio do programa Soil Water Retention Curve - SWRC (Dourado Neto et al., 2001).

Determinou-se, com base nos parâmetros obtidos, o índice $\mathrm{S}$, tangente à curva característica de água no solo no ponto de inflexão, segundo a equação (Dexter, 2004):

$$
\mathrm{S}=-\mathrm{n}(\theta \mathrm{s}-\theta \mathrm{r})\left[1+\frac{1}{\mathrm{~m}}\right]^{-(1+\mathrm{m})}
$$

A resistência do solo à penetração $(\mathrm{RP}), \mathrm{em} \mathrm{kPa}$, foi calculada com base na equação desenvolvida por Dexter et al. (2007) para solos temperados:

$$
\mathrm{RP}=328+37,39\left(\frac{1}{\mathrm{~S}}\right)+1,615 \sigma^{\prime}
$$

em que:

$\sigma$ '- estresse efetivo, em hPa, correspondendo à contribuição da água dos poros para a resistência do solo à penetração

O estresse efetivo foi calculado com base no grau de saturação do solo $(\Theta)$ e na tensão da água dos poros (h) prevalecente:

$$
\sigma^{\prime}=\Theta h
$$

sendo:

$$
\Theta=\frac{(\theta-\theta \mathrm{r})}{(\theta \mathrm{s}-\theta \mathrm{r})}
$$


A tensão da água dos poros foi calculada pela explicitação do h da Eq. 1:

$$
\mathrm{h}(\Theta)=\frac{1}{\alpha}\left[\Theta^{-1 / \mathrm{m}}-1\right]^{1 / \mathrm{n}}
$$

Verifica-se que a equação proposta por Dexter et al. (2007) para estimativa da resistência do solo à penetração é a soma de dois termos simples: um representa o grau de compacidade do solo enquanto o outro representa o efeito da água do solo.

Considerou-se o conteúdo de água do solo correspondente à capacidade de campo $(\theta \mathrm{cc})$ para estimar a resistência do solo à penetração, o qual foi determinado mediante a equação desenvolvida por Andrade \& Stone (2011):

$$
\theta \mathrm{cc}=(\theta \mathrm{s}-\theta \mathrm{r})\left(1-\left(1-\mathrm{p}^{\frac{1-\gamma}{\gamma}}\right)\right)^{\frac{1}{1-\gamma}}+\theta \mathrm{r}
$$

em que:

$\gamma=2,5+[2 /(n-1)]$ e p é uma porcentagem da condutividade hidráulica saturada, no caso considerado igual a 0,01 .

A resistência do solo à penetração foi relacionada ao índice S segundo uma equação do tipo potencial:

$$
\mathrm{S}=\mathrm{cRP}^{\mathrm{d}}
$$

Com base na Eq. 8 foram determinados valores de RP correspondentes a valores de $\mathrm{S}$ citados por Andrade \& Stone (2009) como indicadores de solo de boa qualidade estrutural e de solos inteiramente degradados fisicamente. Os valores obtidos foram comparados com valores de RP tidos como críticos na literatura, para verificar a aplicabilidade da Eq. 3 a solos tropicais.

Valores da densidade do solo foram relacionados com RP para seis classes texturais, muito argilosa, argilosa, argiloarenosa, franco-argilo-arenosa, franco/franco-arenosa e areia-franca/arenosa. Adotando-se os teores médios de argila de 660, 500, 410, 300, 190 e $110 \mathrm{~g} \mathrm{~kg}^{-1}$, respectivamente, para essas classes texturais estabeleceram-se equações potenciais relacionando-se a Ds com o teor de argila para diversos valores de RP. Os valores de Ds obtidos foram confrontados com valores da literatura considerados críticos para diferentes texturas do solo e com os correspondentes valores de RP para verificar, de outra forma, a aplicabilidade da Eq. 3 a solos tropicais.

Ajustaram-se também equações potenciais à RP em função da umidade gravimétrica do solo para as classes texturais argilosa (argila $\left.>350 \mathrm{~g} \mathrm{~kg}^{-1}\right)$, média $\left(350 \mathrm{~g} \mathrm{~kg}^{-1}>\right.$ argila $>150$ $\mathrm{g} \mathrm{kg}^{-1}$ ) e arenosa (argila $\leq 150 \mathrm{~g} \mathrm{~kg}^{-1}$ ) as quais foram comparadas com dados da literatura de umidades consideradas críticas para determinado valor de resistência do solo à penetração, no intuito de também validar a Eq. 3.

Finalmente, compararam-se dados de RP obtidos em campo na Embrapa Arroz e Feijão, em Santo Antônio de Goiás, em 2007 (Cunha et al., 2011) e pelos autores em 2011, usando a mesma metodologia, com os valores estimados pela Eq. 3 .

\section{Resultados e Discussão}

Considerando o valor de $\mathrm{S}=0,045$ como adequado à divisão entre solo de boa qualidade estrutural e solo com tendências a se tornar degradado e $\mathrm{S} \leq 0,025$ como indicativo de solos inteiramente degradados fisicamente (Andrade \& Stone, 2009) verifica-se, pela Figura 1, que esses valores correspondem, respectivamente, a $\mathrm{RP}=1260 \mathrm{kPa}$ e $\mathrm{RP} \geq 1900 \mathrm{kPa}$.

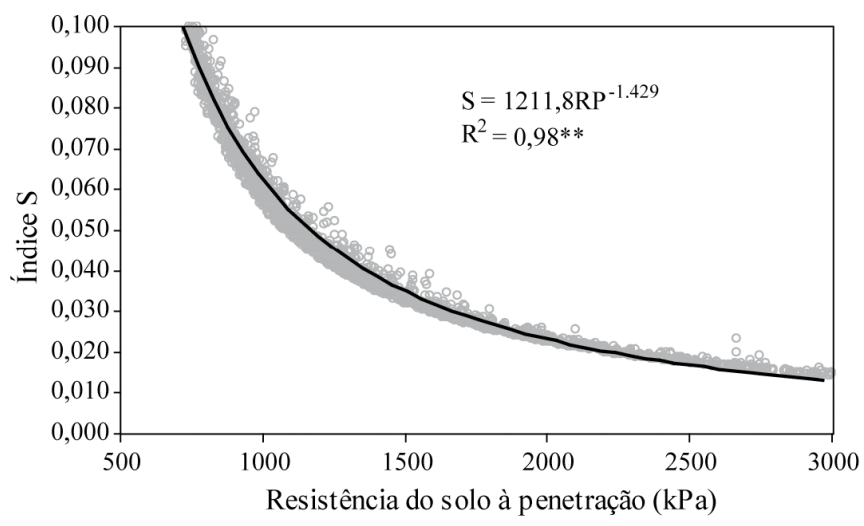

Figura 1. Índice $S$ em função da resistência do solo à penetração (RP) no conteúdo de água no solo correspondente à capacidade de campo

$\mathrm{O}$ valor de $\mathrm{RP}$ correspondente a $\mathrm{S} \leq 0,025$ está próximo de $2000 \mathrm{kPa}$, valor que tem sido considerado limitante ao crescimento e desenvolvimento de sistemas radiculares, por diversos autores (Taylor et al., 1966; Tormena et al., 1998; Lapen et al., 2004), sinalizando que a equação para estimativa de RP proposta por Dexter et al. (2007) pode ser usada para solos tropicais.

Estabelecendo a correspondência entre $\mathrm{S}$ e RP pode-se afirmar que valores de $\mathrm{RP} \leq 1260 \mathrm{kPa}$ indicam ausência de restrição à penetração do solo pelas raízes e valores de $\mathrm{RP} \geq 1900 \mathrm{kPa}$ são indicadores de solos compactados. Esses valores são da mesma ordem de grandeza dos observados por Moreira (2009), que considerou solo com índice S igual a 0,053 como não impeditivo ao crescimento de raízes e com índice $\mathrm{S}$ igual a 0,021 como restritivo a esse crescimento. Pela equação de Dexter et al. (2007) esses índices correspondem, respectivamente, a valores de RP de 1123 e $2147 \mathrm{kPa}$.

A partir das equações ajustadas da Figura 2 foram determinados valores de densidade do solo para RP $=2000$ $\mathrm{kPa}$, em razão dessa RP normalmente corresponder a situações críticas de Ds. Esses valores foram comparados com densidades críticas apresentadas na literatura para diferentes teores de argila (Tabela 1).

Observa-se que os valores calculados de densidade do solo se situaram próximos dos estabelecidos como críticos por Dias Júnior \& Miranda (2000) com base em ensaios de Proctor normal para solos de textura argilosa, média e arenosa (Tabela 1). Apenas para o teor de argila de $370 \mathrm{~g} \mathrm{~kg}^{-1}$ os valores se afastaram mais. Da mesma forma, as densidades calculadas se aproximaram das densidades consideradas críticas por Reinert et al. (2001), Michelon (2005) e Silva et al. (2008).

Apenas na comparação com os dados de Silva (2003) e Almeida et al. (2008) os valores calculados de Ds foram 


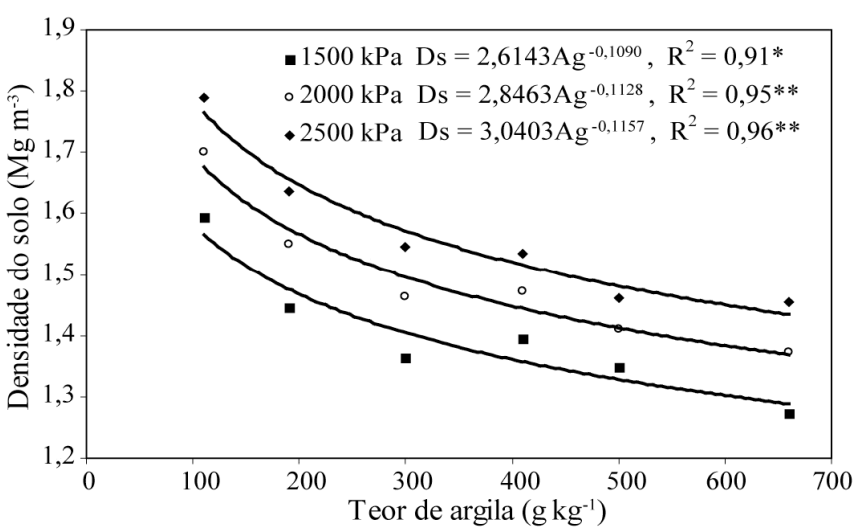

Figura 2. Densidade do solo (Ds) em função do teor de argila (Ag) para diferentes valores de resistência do solo à penetração

Tabela 1. Valores de densidade do solo (Ds) críticos obtidos na literatura para diferentes teores de argila e calculados pela fórmula da Figura 2 referente à resistência do solo à penetração de 2000 kPa

\begin{tabular}{|c|c|c|c|}
\hline $\begin{array}{l}\text { Argila } \\
\left(\mathrm{g} \mathrm{kg}^{-1}\right)\end{array}$ & $\begin{array}{c}\text { Ds literatura } \\
\left(\mathrm{Mg} \mathrm{m}^{-3}\right)\end{array}$ & Referência & $\begin{array}{c}\text { Ds calculada } \\
\left(\mathrm{Mg} \mathrm{m}^{-3}\right)\end{array}$ \\
\hline 570 & 1,43 & Dias Júnior \& Miranda (2000) & 1,39 \\
\hline 370 & 1,57 & Dias Júnior \& Miranda (2000) & 1,46 \\
\hline 300 & 1,53 & Dias Júnior \& Miranda (2000) & 1,50 \\
\hline 130 & 1,64 & Dias Júnior \& Miranda (2000) & 1,64 \\
\hline$>550$ & 1,45 & Reinert et al. (2001) & 1,40 \\
\hline $200-550$ & 1,55 & Reinert et al. (2001) & $1,57-1,40$ \\
\hline$<200$ & 1,65 & Reinert et al. (2001) & $1,69 *-1,57$ \\
\hline 600 & 1,53 & Silva (2003) & 1,38 \\
\hline 500 & 1,62 & Silva (2003) & 1,41 \\
\hline $0-200$ & 1,60 & Michelon (2005) & $1,69 *-1,57$ \\
\hline $200-300$ & 1,55 & Michelon (2005) & $1,57-1,50$ \\
\hline $300-400$ & 1,50 & Michelon (2005) & $1,50-1,45$ \\
\hline $400-500$ & 1,45 & Michelon (2005) & $1,45-1,41$ \\
\hline $500-600$ & 1,40 & Michelon (2005) & $1,41-1,38$ \\
\hline 120 & 1,70 & Almeida et al. (2008) & 1,66 \\
\hline 310 & 1,54 & Almeida et al. (2008) & 1,49 \\
\hline 220 & $1,46-1,98$ & Silva et al. (2008) & 1,55 \\
\hline 310 & $1,40-1,79$ & Silva et al. (2008) & 1,49 \\
\hline 390 & $1,38-1,70$ & Silva et al. (2008) & 1,45 \\
\hline 480 & $1,36-1,64$ & Silva et al. (2008) & 1,42 \\
\hline 580 & $1,34-1,58$ & Silva et al. (2008) & 1,39 \\
\hline
\end{tabular}

*Teor de argila menor que $200 \mathrm{~g} \mathrm{~kg}^{-1}$ ou no intervalo de 0 a $200 \mathrm{~g} \mathrm{~kg}^{-1}$ foi considerado como sendo $100 \mathrm{~g} \mathrm{~kg}^{-1}$

menores. Silva (2003) observou reduções na produtividade do trigo em solo muito argiloso e argiloso com densidades do solo de, respectivamente, 1,53 e $1,62 \mathrm{Mg} \mathrm{m}^{-3}$. Almeida et al. (2008) estabeleceram, após compactação do solo em laboratório, as densidades de 1,70 e $1,54 \mathrm{Mg} \mathrm{m}^{-3}$ como críticas, respectivamente, para solos de textura arenosa e argilosa.

Ao se ajustar equações potenciais à RP em função da umidade gravimétrica (Figura 3) verificou-se, para o valor de $\mathrm{RP}=2000 \mathrm{kPa}$, que os teores de umidade considerados críticos, foram 0,$074 ; 0,177$ e $0,225 \mathrm{~kg} \mathrm{~kg}^{-1}$, respectivamente, para solo de textura arenosa, média e argilosa.

Santana et al. (2006) encontraram, considerando a resistência do solo à penetração de $2000 \mathrm{kPa}$, valores entre 0,020 a $0,110 \mathrm{~kg} \mathrm{~kg}^{-1}$ como umidades consideradas críticas para quatro horizontes arenosos e de 0,110 a $0,170 \mathrm{~kg} \mathrm{~kg}^{-1}$ para

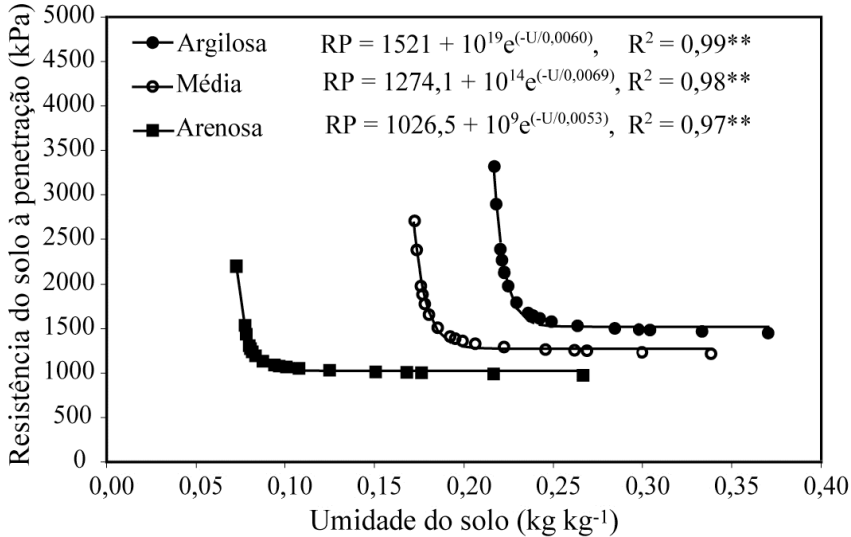

Figura 3. Resistência do solo à penetração (RP) em função da umidade gravimétrica $(U)$ para solos de textura argilosa, média e arenosa

solos de textura média. Para o mesmo valor de RP Silveira et al. (2010) verificaram que o intervalo de umidade crítica se situou entre 0,062 e $0,096 \mathrm{~kg} \mathrm{~kg}^{-1}$ para solo arenoso. Almeida et al. (2008) constataram, após compactação de solo em laboratório, variações de umidade de 0,020 a $0,140 \mathrm{~kg} \mathrm{~kg}^{-1} \mathrm{e}$ de 0,140 a $0,320 \mathrm{~kg} \mathrm{~kg}^{-1}$, respectivamente, para solos arenosos e argilosos. Dias Júnior \& Miranda (2000) também encontraram, em laboratório, umidades ótimas para compactação de 0,200 a $0,300 \mathrm{~kg} \mathrm{~kg}^{-1}$ para solos argilosos. Com o uso das equações estabelecidas por Silva et al. (2008) para cálculo de RP em função da umidade e densidade do solo, se encontraram as umidades críticas de 0,132 a $0,184 \mathrm{~kg} \mathrm{~kg}^{-1}$ para solos de textura média e de 0,225 a $0,309 \mathrm{~kg} \mathrm{~kg}^{-1}$ para solos de textura argilosa, para $\mathrm{RP}=2000 \mathrm{kPa}$. Ao confrontar todos esses dados com os limites críticos de umidade do solo estabelecidos a partir das equações apresentadas na Figura 3, constata-se que eles estão dentro ou muito próximos dos intervalos de umidade medidos por esses diversos autores, o que fortalece a possibilidade de se utilizar, em solos tropicais, a equação para estimativa de RP proposta por Dexter et al. (2007).

$\mathrm{Na}$ comparação dos dados observados em campo com os estimados pela Eq. 3 verificou-se que essa equação subestimou os valores observados em cerca de $10 \%$; contudo e de acordo com o coeficiente de determinação $\left(\mathrm{R}^{2}\right)$, ela foi capaz de explicar

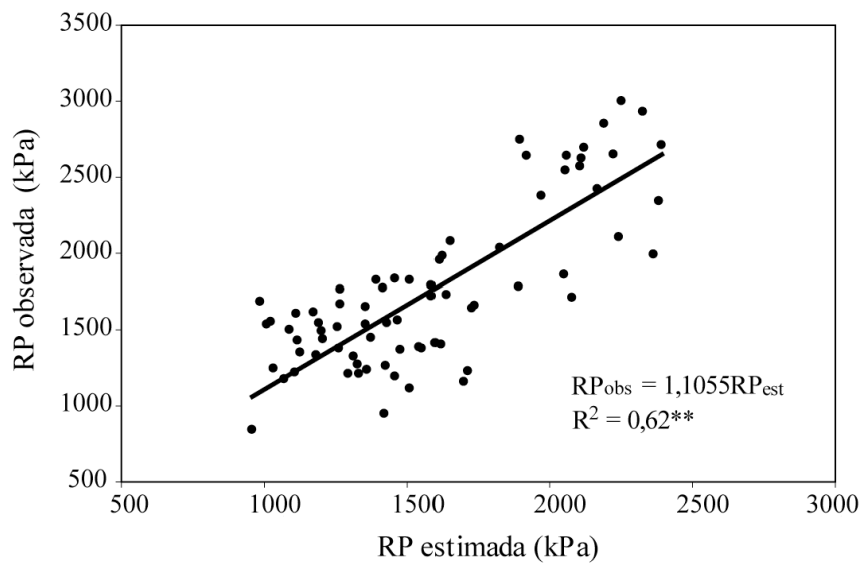

Figura 4. Relação entre a resistência do solo à penetração (RP) estimada e a observada 
$62 \%$ da variabilidade da RP. É interessante que valores de RP obtidos com a equação proposta sejam comparados com dados de campo de uma grande faixa de classes e texturas de solo.

$\mathrm{O}$ valor de $\mathrm{R}^{2}$ não foi maior devido, possivelmente, à variação espacial das propriedades do solo. A resistência à penetração e a umidade foram medidas no campo enquanto as características de retenção de água foram determinadas utilizando-se diferentes amostras de solo indeformadas. Adicionalmente, valores da tensão da água dos poros, $\mathrm{h}(\Theta)$, são estimados a partir dos conteúdos medidos da água do solo em combinação com a forma invertida da equação de Genuchten (1980) (Eq. 6), cujos parâmetros também estão associados a erros de medida e variabilidade espacial.

\section{CONCLUSÕES}

1. Na região do cerrado valores de resistência do solo à penetração iguais ou maiores que $1900 \mathrm{kPa}$, determinados no conteúdo de água do solo equivalente à capacidade de campo, podem ser considerados indicadores de solos compactados.

2. A equação proposta por Dexter et al. (2007) para estimativa da resistência do solo à penetração é adequada para os solos do cerrado, embora deva ser mais testada para determinar seu poder de predição.

\section{Agradecimentos}

Os autores agradecem ao ex-pesquisador da Embrapa Cerrados, Dr. Euzébio Medrado da Silva, pelas informações referentes às análises fisico-hídricas de amostras de solo armazenadas no banco de dados dessa instituição.

\section{Literatura Citada}

Almeida, C. X.; Centurion, J. F.; Freddi, O. da S.; Jorge, R. F.; Barbosa, J. C. Funções de pedotransferência para a curva de resistência à penetração. Revista Brasileira de Ciência do Solo, v.32, p.2235-2243, 2008.

Andrade, R. da S.; Stone, L. F. Índice S como indicador da qualidade física de solos do cerrado brasileiro. Revista Brasileira de Engenharia Agrícola e Ambiental, v.13, p.382388, 2009.

Andrade, R. da S.; Stone, L. F. Estimativa da umidade na capacidade de campo em solos sob cerrado. Revista Brasileira de Engenharia Agrícola e Ambiental, v.15, p.111-116, 2011.

Busscher, W. J.; Bauer, P. J.; Camp, C. R.; Sojka, R. E. Correction of cone index for soil water content differences in a Coastal Plain soil. Soil \& Tillage Research, v.43, p.205-217, 1997.

Cunha, E. de Q.; Stone, L. F.; Moreira, J. A. A.; Ferreira, E. P. de B.; Didonet, A. D.; Leandro, W. M. Sistemas de preparo do solo e culturas de cobertura na produção orgânica de feijão e milho. I - Atributos físicos do solo. Revista Brasileira de Ciência do Solo, v.35, p.589-602, 2011.

Dexter, A. R. Soil physical quality. Part I. Theory, effects of soil texture, density, and organic matter, and effects on root growth. Geoderma, v.120, p.201-214, 2004.

Dexter, A. R.; Czyz, E. A.; Gate, O. P. A method for prediction of soil penetration resistance. Soil \& Tillage Research, v.93, p.412-419, 2007.
Dias Júnior, M. S.; Miranda, E. E. V. Comportamento da curva de compactação de cinco solos da região de Lavras (MG). Ciência e Agrotecnologia, v.24, p.337-346, 2000.

Dourado Neto, D.; Nielsen, D. R.; Hopmans, J. W.; Reichardt, K.; Bacchi, O. O. S.; Lopes, P. P. Programa para confecção da curva de retenção de água no solo, modelo van Genuchten. Soil water retention curve, SWRC (version 3,00 beta). Piracicaba: USP, 2001.

Genuchten, M. Th. van. A closed-form equation for predicting the hydraulic conductivity of unsaturated soils. Soil Science Society of America Journal, v.44, p.892-898, 1980.

Imhoff, S; Silva, A. P; Tormena, C. A. Aplicações da curva de resistência no controle da qualidade física de um solo sob pastagem. Pesquisa Agropecuária Brasileira, v.35, p.1493$1500,2000$.

Lapen, D. R.; Topp, G. C.; Gregorich, E. G.; Curnoe, W. E. Least limiting water range indicators of soil quality and corn production, Eastern Ontario, Canadá. Soil \& Tillage Research, v.78, p.151-170, 2004.

Michelon, C. J. Qualidade física dos solos irrigados do Rio Grande do Sul e do Brasil Central. Santa Maria: UFSM, 2005. 92p. Dissertação Mestrado

Moreira, N. B. Qualidade física de um Argissolo Amarelo em áreas cultivadas com laranja no nordeste paraense. Belém: UFRA, 2009. 55p. Dissertação Mestrado

Ralisch, R; Miranda, T. M.; Okumura, R. S.; Barbosa, G. M. de C.; Guimarães, M. de F.; Scopel, E.; Balbino, L. C. Resistência à penetração de um Latossolo Vermelho Amarelo do Cerrado sob diferentes sistemas de manejo. Revista Brasileira de Engenharia Agrícola e Ambiental, v.12, p.381-384, 2008.

Reinert, D. J.; Reichert, J. M.; Silva, V. R. Propriedades físicas de solos em sistema de plantio direto irrigado. In: Carlesso, R.; Petry, M. T.; Rosa, G. M.; Ceretta, C.A. (ed.). Irrigação por aspersão no Rio Grande do Sul. Santa Maria: Imprensa Universitária, 2001. p.114-133.

Ribon, A. A.; Tavares Filho, J. Models for the estimation of the physical quality of a Yellow Red Latosol (Oxisol) under pasture. Brazilian Archives of Biology and Technology, v.47, p.25-31, 2004.

Sá, M. A. C.; Santos Júnior, J. D. G. Compactação do solo: conseqüências para o crescimento vegetal. Planaltina: Embrapa Cerrados, 2005. 26p. Documentos, 136

Santana, M. B.; Souza, L. da S.; Souza, L. D.; Fontes, L. E. F. Atributos físicos do solo e distribuição do sistema radicular de citros como indicadores de horizontes coesos em dois solos de tabuleiros costeiros do estado da Bahia. Revista Brasileira de Ciência do Solo, v.30, p.1-12, 2006.

Silva, A. P.; Tormena, C. A.; Fidalski, J.; Inhoff, S. Funções de pedotransferência para as curvas de retenção de água e de resistência do solo à penetração. Revista Brasileira de Ciência do Solo, v.32, p.1-10, 2008.

Silva, V. R. Propriedades físicas e hídricas em solos sob diferentes estados de compactação. Santa Maria: UFSM, 2003. 171p. Tese Doutorado

Silveira, D. C.; Melo Filho, J. F.; Sacramento, J. A. A. S.; Silveira E. C. P. Relação umidade versus resistência à penetração para um Argissolo Amarelo distrocoeso no recôncavo da Bahia. Revista Brasileira de Ciência do Solo, v.34, p.659-667, 2010. 
Taylor, H. M.; Roberson, G. M.; Parker Júnior, J. J. Soil strength-root penetration relations for medium- to coarsetextured soil materials. Soil Science, v.102, p.18-22, 1966.

Tormena, C. A.; Friedrich, R.; Pintro, J. C.; Costa, A. C. S.; Fidalski, J. Propriedades físicas e taxa de estratificação de carbono orgânico num Latossolo Vermelho após dez anos sob dois sistemas de manejo. Revista Brasileira de Ciência do Solo, v.28, p.1023-1031, 2004.
Tormena, C. A.; Roloff, G.; Sá, J. C. M. Propriedades físicas do solo sob plantio direto influenciadas por calagem, preparo inicial e tráfego. Revista Brasileira de Ciência do Solo, v.22, p.301-309, 1998.

Whalley, W. R.; Harrison, P. B. L.; Clark, L. J.; Gowing, D. J. G. Use of effective stress to predict the penetrometer resistance of unsaturated agricultural soils. Soil \& Tillage Research, v.84, p.18-27, 2005. 